\title{
Evaluation of perinatal arterial ischemic stroke using noninvasive arterial spin labeling perfusion MRI
}

\author{
Jill B De Vis' ${ }^{1}$ Esben T Petersen' ${ }^{1}$, Karina J Kersbergen ${ }^{2}$, Thomas Alderliesten², Linda S de Vries ${ }^{2}$, Frank van Bel ${ }^{2}$,
} Floris Groenendaal ${ }^{2}$, Petra MA Lemmers ${ }^{2}$, Jeroen Hendrikse' and Manon JNL Benders ${ }^{2}$

BACKGROUND: Arterial spin labeling (ASL) magnetic resonance imaging (MRI) can evaluate brain perfusion in neonates noninvasively. The aim of this study was to investigate whether ASL MRI can demonstrate perfusion abnormalities in neonates diagnosed with perinatal arterial ischemic stroke (PAIS).

METHODS: Pulsed ASL perfusion MR images were acquired in the subacute stage (5-6 d after birth) and at follow-up (13 d to 16 wk after birth) in four PAIS patients. Images were visually evaluated for hypo- and hyperperfusion. In addition, cerebral oxygenation was monitored using near infrared spectroscopy (NIRS).

RESULTS: In three PAIS patients, ASL images showed hypoperfusion in the stroke area. In one of these, hyperperfusion was visualized in the periphery of the stroke area. In one PAIS patient, hyperperfusion was seen in the stroke area. In all infants, cerebral oxygenation was higher in the infarcted hemisphere as compared with the contralateral hemisphere. Follow-up ASL images showed partial recovery of perfusion in the stroke area.

CONCLUSION: ASL perfusion MRI is able to reliably detect hypo- and hyperperfusion in PAIS patients and can be used to monitor the evolution of perfusion after an ischemic event.

P erinatal arterial ischemic stroke (PAIS) has an estimated incidence of 1 in 2,300 live births (1). Most often PAIS presents with convulsions. Initial assessment is performed with (Doppler) ultrasound and amplitude-integrated electroencephalography (aEEG). Magnetic resonance imaging (MRI) is used to confirm PAIS. Although neonatal MRI enables prediction of motor outcome in PAIS patients by localizing the stroke area according to the posterior limb of the internal capsule or by visualizing pre-Wallerian degeneration $(2,3)$, it remains difficult to determine the viability of brain tissue after stroke. In adults it has been shown that arterial spin labeling (ASL) perfusion MRI, a noninvasive technique, is valuable for the assessment of acute stroke $(4,5)$. Perfusion deficits and perfusion-diffusion mismatches as visualized on the ASL images have been proven to correspond with dynamic susceptibility contrast-enhanced images (4). Furthermore, a relation between perfusion in the affected hemisphere and outcome has been shown (5). Although noninvasive ASL perfusion imaging seems to be a promising tool in the neonatal population, experience is still limited (6-11), and only one study has described the use of ASL MRI in the evaluation of PAIS (10). In this study, it was shown that ASL MRI could demonstrate areas of hypo- and hyperperfusion in patients with PAIS. However, ASL has not yet been used to assess changes in perfusion (abnormalities) over time in neonates with stroke. The aim of this study was to compare initial ASL perfusion MR images to images obtained at follow-up. For this purpose, ASL MR images of four PAIS patients were evaluated. In addition, regional cerebral oxygenation $\left(\mathrm{rScO}_{2}\right)$ was monitored, compared with reported $\mathrm{rScO}_{2}$ values in neonates $(63 \pm 12 \%$ $(12,13))$, and analyzed as a function of the perfusion signal as visualized on the ASL MR images.

\section{RESULTS}

\section{Case 1}

Characteristics of patient 1 are shown in Table 1. This patient presented with central apnea $2 \mathrm{~d}$ after birth. Single-channel aEEG and subsequent conventional EEG showed unilateral, left-sided epileptic activity during apneas. Following administration of phenobarbitone $(20 \mathrm{mg} / \mathrm{kg})$, no further clinical or electrographic seizures (on aEEG) were seen. After stroke was diagnosed in the territory of the left medial cerebral artery (MCA) on computed tomography, the patient was transferred from another academic hospital to our institution. In our institution, aEEG showed asymmetry in background activity, with more discontinuous background activity on the affected side as compared with the continuous activity $(\mathrm{CNV})$ on the nonaffected side. No electrographic discharges (on aEEG) or clinical seizures were detected in our hospital. MRI (on a $1.5 \mathrm{~T}$ MRI scanner) showed occlusion of the ipsilateral MCA on the time-of-flight (TOF) images (Figure 1a). Flow volume measurements with two-dimensional phase-contrast MR angiography (PC-MRA) showed a higher flow in the ipsilateral internal carotid artery as compared with the contralateral internal carotid artery (Table 2). Bedside monitoring using near infrared spectroscopy (NIRS) showed higher $\mathrm{rScO}_{2}$ values in the ipsilateral hemisphere as compared with the contralateral 


\section{Articles $\mid$ De Vis et al.}

Table 1. Patient characteristics

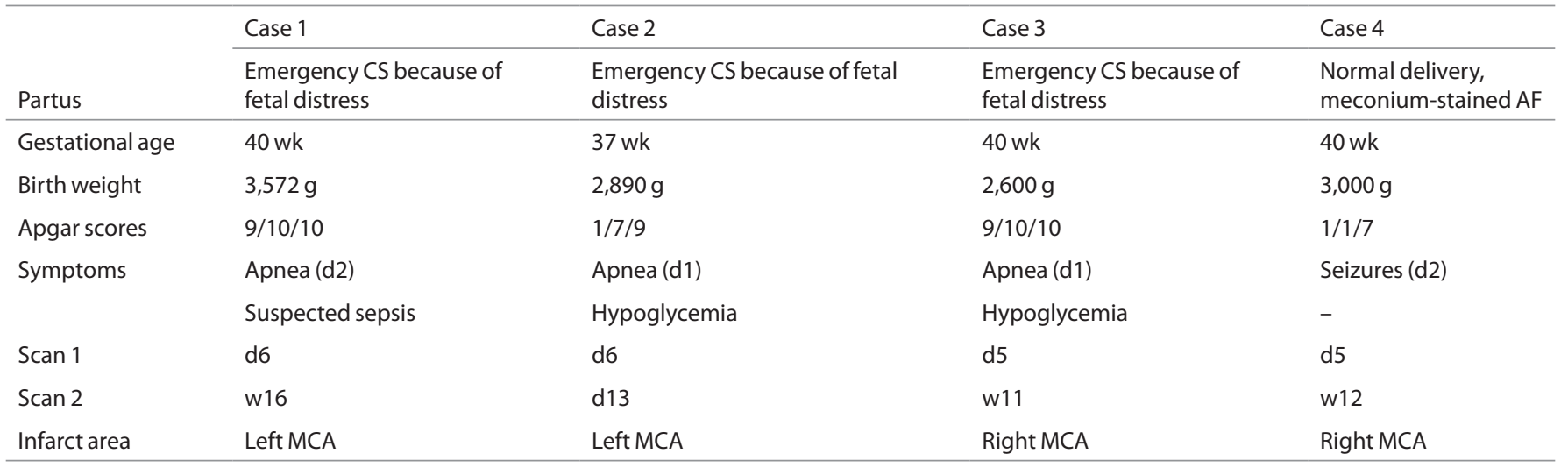

AF, amniotic fluid; CS, cesarean section; d, day; MCA, medial cerebral artery; MR, magnetic resonance imaging; w, week.

Gestational age shows the postconceptional age of the neonates at birth. The symptoms that raised the suspicion for perinatal arterial ischemic stroke (PAIS) are given, as well as on which day they presented themselves. In addition, clinical symptoms possibly related to PAIS, which were detected in our neonates, are shown. Scan 1 shows on which day after birth the initial scan was performed. Scan 2 denotes on which day or how many weeks after birth follow-up MRI was obtained.

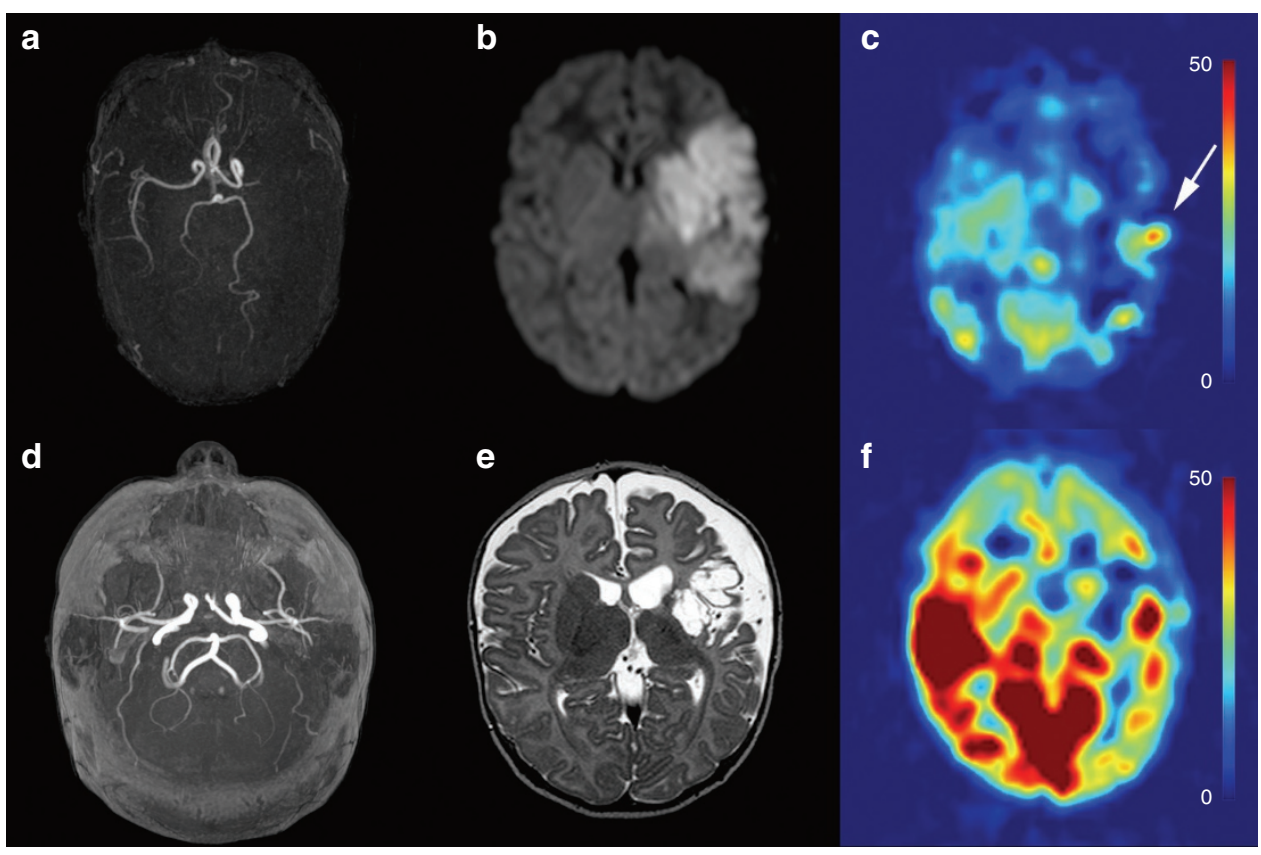

Figure 1. MRI of case 1. (a-c) MR images obtained on the sixth day. (a) TOF MR image (1.5 T) shows occlusion of the left medial cerebral artery (MCA). (b) Diffusion restriction in the supply territory of the left MCA. (c) ASL image shows lower perfusion in the left hemisphere corresponding with the diffusion-restricted area. A higher perfusion with an arterial transit artifact (arrow) is visualized in the periphery of the stroke area. (d-f) MR images obtained at week 16. (d) Flow seen on the TOF image appears to be normal. (e) T2-weighted image, tissue loss in the territory of the left MCA. (f) ASL image, area of lower perfusion extending from the external capsule and basal ganglia on the left side toward the frontal cortex. As compared with c, the initial ASL images, the region with lower perfusion signal seems to be smaller. ASL, arterial spin labeling; MR, magnetic resonance; TOF, time-of-flight.

hemisphere (Figure 2). MRI was repeated at day 6 after birth on a 3 T MRI scanner. Diffusion restriction was shown in the territory of the ipsilateral MCA (Figure 1b). Corresponding with this area, a lower perfusion signal was seen on the ASL images (Figure 1c). In the cortex of the ipsilateral hemisphere, higher perfusion was seen, with a bright spot representing delayed arrival of transit (Figure 1c). Follow-up MRI performed $16 \mathrm{wk}$ after birth showed normal flow in the ipsilateral MCA on the TOF images (Figure 1d). The T2-weighted images showed tissue loss in the external capsule, basal ganglia, and frontal region on the ipsilateral side (Figure 1e). Whole-brain perfusion measured on the ASL images was increased as compared with the earlier images (Table 2). When visually comparing the two ASL images, perfusion seemed to be restored partly in the stroke area. Still, a lower perfusion signal was seen in the area corresponding with the external capsule, basal ganglia, and the frontal cortex (Figure 1f).

\section{Case 2}

Characteristics of patient 2 are shown in Table 1. This patient was born in another level three hospital. On day 1 , convulsions were clinically suspected and confirmed with aEEG and conventional 
Table 2. Perfusion and volume flow

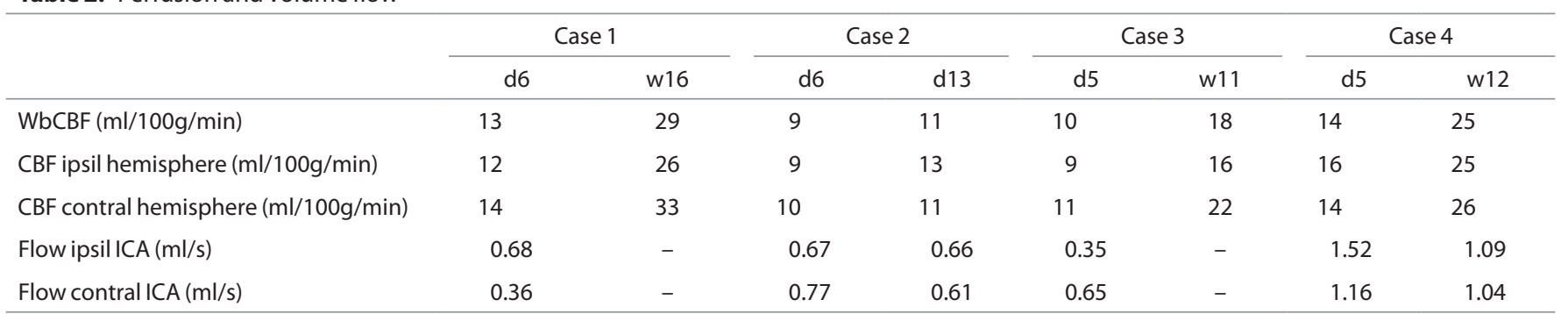

CBF in the hemisphere ipsilateral (ipsil) to the stroke area and in the hemisphere contralateral (contral) to the stroke area are shown. Volume flow of the ipsil ICA and of the contral ICA are shown. In cases 1 and 3, volume flow measurements were not performed at follow-up.

CBF, cerebral blood flow; d, day; ICA, internal carotid artery; w, week; WbCBF, whole-brain cerebral blood flow.

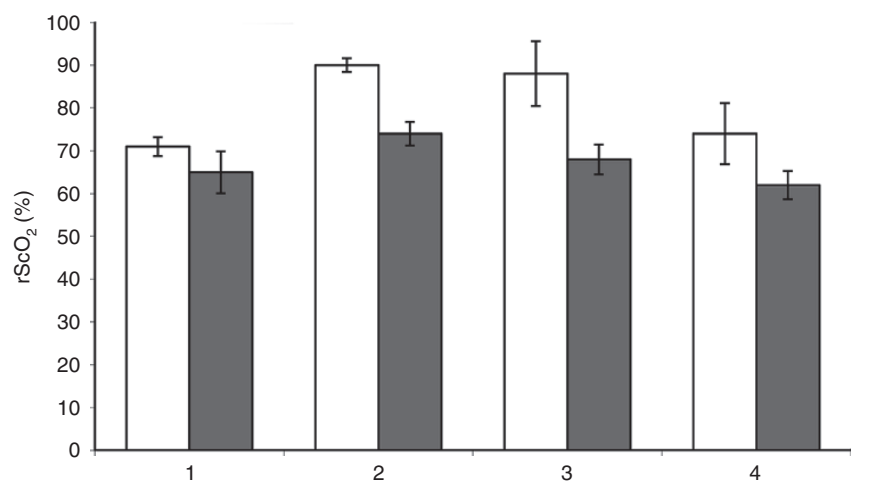

Figure 2. Regional cerebral oxygen saturation $\left(\mathrm{rScO}_{2}\right)$ was measured with near infrared spectroscopy measurements in the ipsilateral and contralateral hemispheres. Measured values obtained half an hour before and after MRI were averaged and are shown. The white columns show values for each case obtained in the ipsilateral hemisphere (mean $\pm S D$ ). The gray columns show values for each case obtained in the contralateral hemisphere (mean $\pm \mathrm{SD}$ ).

EEG. These showed left-sided epileptic activity during apneas, which was successfully treated with phenobarbitone $(20 \mathrm{mg} / \mathrm{kg})$ on day 2 after birth. The infant required ventilation but was extubated on day 3 , no longer requiring ventilatory support. On day 4 the patient was transferred to our institution to participate in a clinical trial. Bedside monitoring with NIRS showed an increased $\mathrm{rSCO}_{2}$ in the ipsilateral hemisphere (Figure 2); aEEG monitoring showed a left-right difference in background pattern, with a discontinuous background activity pattern on the affected side, as compared with a CNV pattern with sleep-wake cycling on the nonaffected side. No clinical or subclinical seizures were seen following admission to our neonatal intensive care unit. MRI performed on day 6 showed a narrow and irregular left MCA on the TOF images (Figure 3a); the M1 segment of the MCA was visible only on the source images. Volume flow measured with PC-MRA showed comparable flow through both internal carotid arteries (Table 2). Diffusion restriction was shown on diffusion-weighted images (Figure 3b). In addition, ASL images showed a lower perfusion signal in the ipsilateral MCA territory as compared with the contralateral MCA territory (Figure 3c). Follow-up MRI performed on day 13 showed no change in the configuration of the ipsilateral MCA (Figure 3d). The T2-weighted images showed a clear delineation of the infarct area (Figure 3e). An area of lower perfusion was visualized in the ipsilateral hemisphere as compared with the contralateral hemisphere. In the periphery of the infarcted region, higher perfusion, with delayed arrival of transit and arterial transit artifacts, was visualized (Figure 3f). Whole-brain perfusion was increased as compared with the earlier ASL images (Table 2).

\section{Case 3}

Characteristics of patient 3 are shown in Table 1. Within $8 \mathrm{~h}$ after birth, this baby presented with apneas and smacking. Because of suspected seizures, the baby was treated with phenobarbitone $(20 \mathrm{mg} / \mathrm{kg})$ and transferred from a level two hospital to our level three hospital. Immediately after admission, aEEG confirmed electrographic discharges from the right hemisphere. The baby received additional antiepileptic medications (lidocaine and midazolam), Thereafter, no clinical or electrographic seizures were detected and aEEG showed a difference in the background pattern, with a discontinuous background activity pattern on the affected side as compared with a CNV pattern with sleep-wake cycling on the nonaffected side. Because of the sedative effect of antiepileptic drugs, the infant needed intubation and ventilation. MRI performed on day 2 (1.5 T MRI scanner) confirmed stroke in the right MCA area. A reduced filling of the ipsilateral MCA was seen on the TOF images (Figure 4a). Two days later, monitoring with aEEG showed a CNV pattern with sleep-wake cycling in both hemispheres, and monitoring using NIRS showed an increased $\mathrm{rScO}_{2}$ in the ipsilateral hemisphere (Figure 2). MRI on a $3 \mathrm{~T}$ MRI scanner performed on day 5 showed diffusion restriction in the territory of the ipsilateral MCA (Figure 4b), and volume flow, evaluated with PC-MRA, was lower toward the ipsilateral hemisphere (Table 2). ASL images visualized a lower perfusion in the supply territory of the ipsilateral MCA (Figure 4c). Flow through the ipsilateral MCA appeared to be normal on follow-up MRI performed $11 \mathrm{wk}$ after birth (Figure 4d). The T2-weighted images showed tissue loss and cystic evolution of the infarct area (Figure 4e). Whole-brain perfusion measured on the ASL images showed an overall higher perfusion as compared with the initial ASL images (Table 2). A lower perfusion was seen in the ipsilateral hemisphere, corresponding with the area of tissue loss (Figure 4f).

\section{Case 4}

Characteristics of patient 4 are shown in Table 1. This infant developed hemiconvulsions of the left arm and leg on day 2 


\section{Articles $\mid$ De Vis et al.}

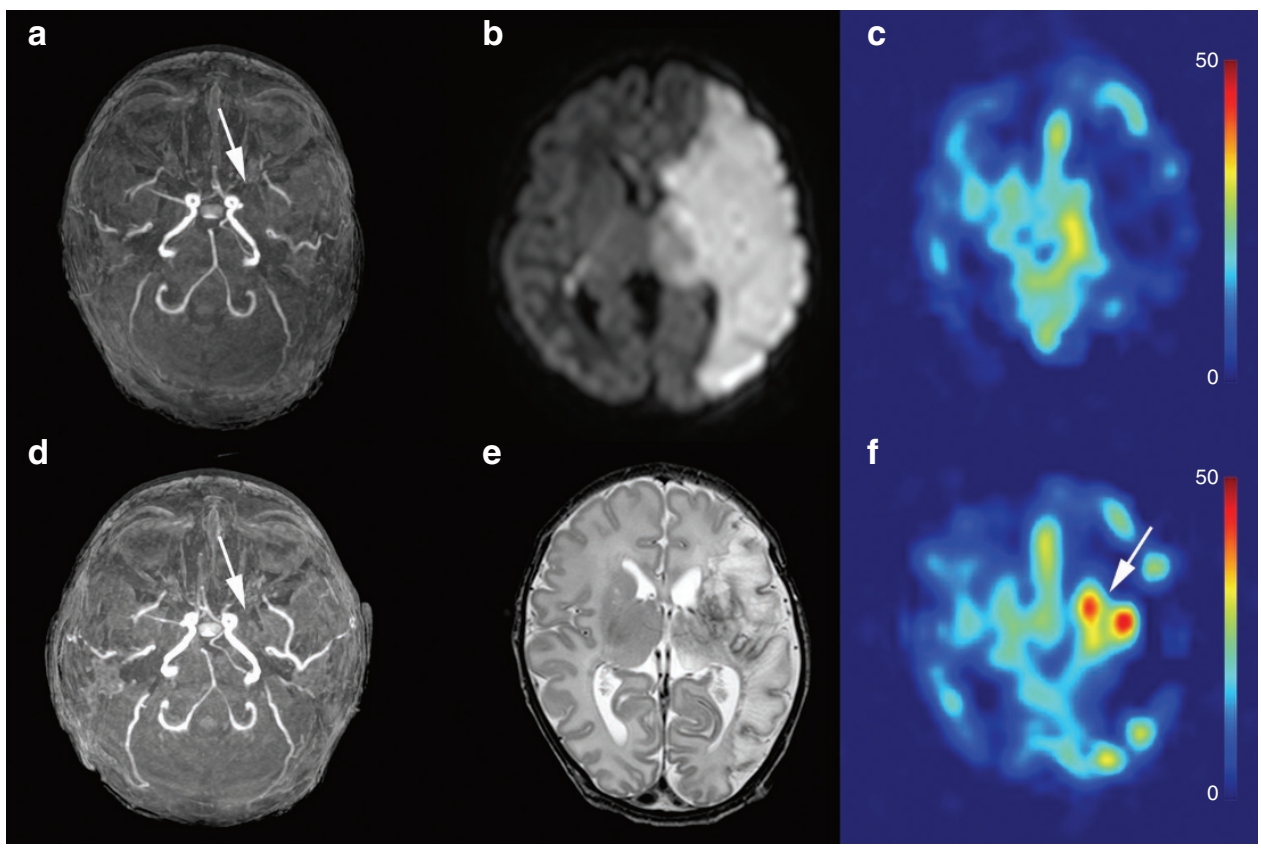

Figure 3. MRI of case 2. (a-c) MR images made at day 6. (a) TOF image, narrow left MCA (arrow). (b) A diffusion-restricted area is shown in the territory of the left MCA. (c) A lower perfusion is seen in the territory of the left MCA on the ASL images. (d-f) MR images made at day 13. (d) TOF image, narrow left MCA (arrow). (e) The stroke area visualized on a T2-weighted image. (f) A region of higher perfusion with a bright spot-an arterial transit artifact-is shown on the ASL images (arrow). ASL, arterial spin labeling; MCA, medial cerebral artery; MR, magnetic resonance; TOF, time-of-flight.

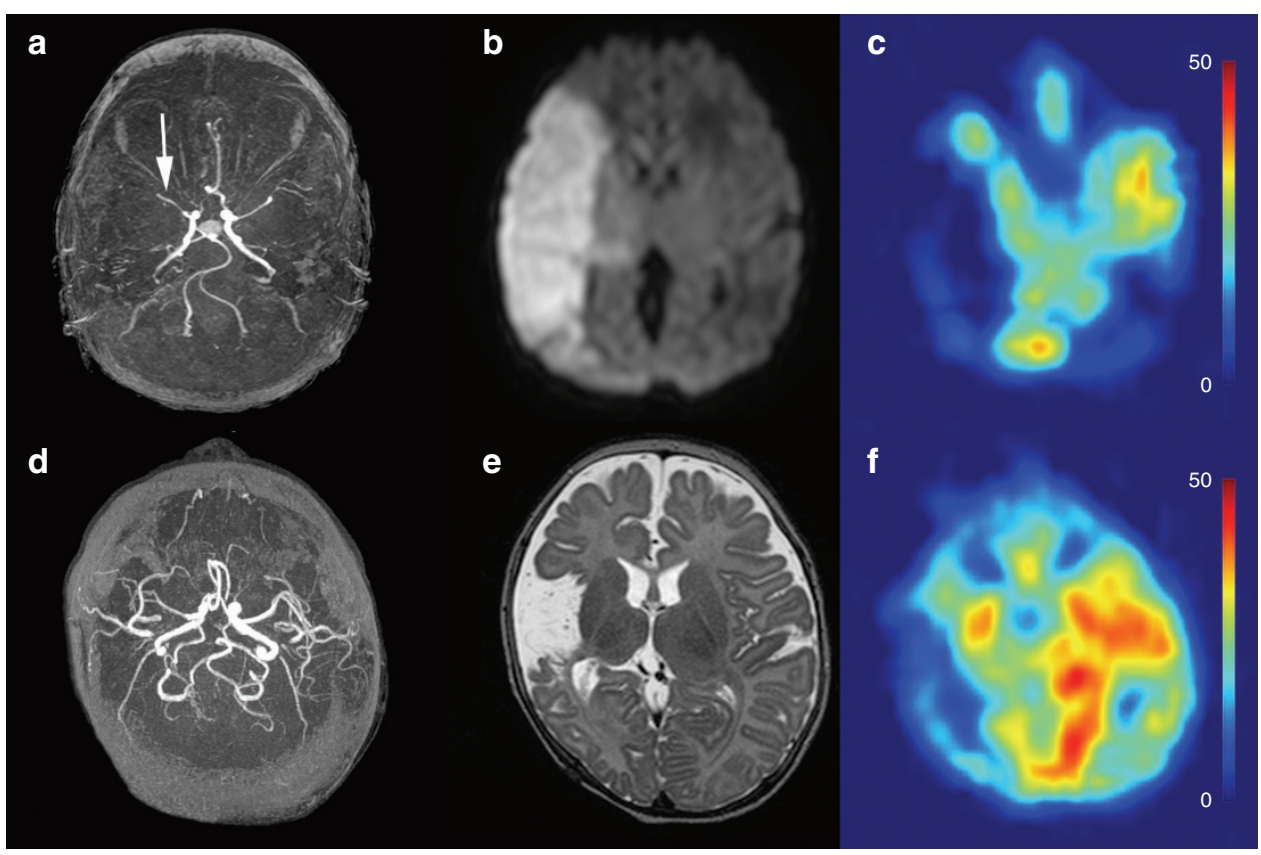

Figure 4. MRI of case 3. (a-c) Images obtained at day 5. (a) Normal flow through the right MCA (arrow). (b) Diffusion restriction in the right MCA territory. (c) ASL MR image shows a lower perfusion in the territory of the right MCA. (d-f) Images obtained at week 11. (d) Normal flow in the right MCA. (e) T2-weighted image, showing tissue loss in the right MCA territory. (f) ASL image, showing lower perfusion in the stroke area. ASL, arterial spin labeling; MCA, medial cerebral artery; MR, magnetic resonance.

after birth. Convulsions in the right hemisphere were confirmed with a single-channel aEEG and with conventional EEG. This was successfully treated with phenobarbitone. On cranial ultrasound, an area of increased echogenicity was recognized, after which the baby was referred to our hospital on day 4 for additional neuro-imaging to confirm PAIS of the right hemisphere. In our institution, neurological examination no longer showed any epileptic activity, and a normal $\mathrm{CNV}$ background without any asymmetries was seen on aEEG. Monitoring with NIRS showed increased $\mathrm{rScO}_{2}$ in the ipsilateral hemisphere (Figure 2);TOF MRI (3 T), performed on day 5, showed normal flow (Figure 5a); and volume flow, 


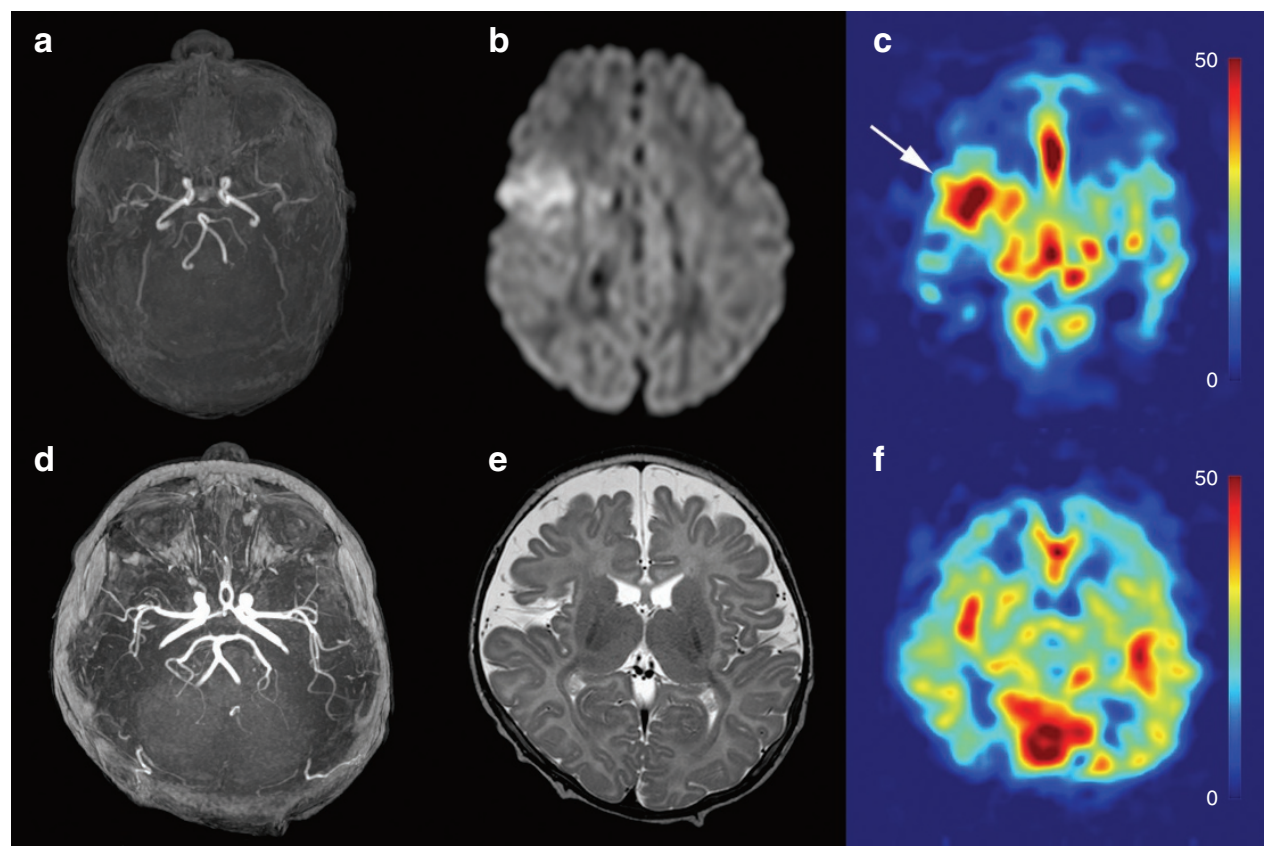

Figure 5. MRI of case 4. (a-c) MR images obtained at day 5. (a) Normal flow through the right MCA. (b) Diffusion restriction in the territory of the right MCA. (c) ASL image shows a hyperperfused area in the right MCA territory corresponding with the area of diffusion restriction (arrow). (d-f) Images obtained at week 12. (d) Normal flow through the right MCA. (e) A wider Sylvian fissure and infarction can be visualized on the T2-weighted images. (f) ASL MRI shows slightly lower perfusion in the stroke region. ASL, arterial spin labeling; MCA, medial cerebral artery; MR, magnetic resonance.

measured with PC-MRA, showed increased flow toward the ipsilateral hemisphere. Diffusion restriction was shown from the right insula toward the frontal cortex on the diffusionweighted images, which confirmed stroke in the right MCA territory (Figure 5b). ASL imaging showed hyperperfusion (arrow) in the area corresponding with the area of diffusion restriction (Figure 5c). Follow-up MRI was performed $12 \mathrm{wk}$ after birth. Flow through the ipsilateral MCA appeared to be normal (Figure 5d). On the T2-weighted images a wider Sylvian fissure, due to tissue loss in the frontal area, was visualized in the right hemisphere, and infarction was visible in the gray and white matter (Figure 5e). A slightly lower perfusion was visualized on the ASL images in the region corresponding with the infarcted area (Figure 5f).

\section{DISCUSSION}

Arterial spin labeling MR images of four PAIS patients were analyzed. ASL MRI was able to denote areas of hypo- and hyperperfusion in correspondence with the stroke area as visualized on the diffusion-weighted images. In addition, arterial transit artifacts, which represent delayed arrival of labeled signal through collateral arteries, were visualized and followup ASL MR images showed partial recovery of perfusion in the stroke area. Our findings are in agreement with a previously performed study that analyzed ASL images of four PAIS patients (10). In this study, hypoperfusion was visualized in the center of the stroke area and a higher perfusion signal was seen in the periphery of the stroke. This is similar to our finding of a higher perfusion signal in the periphery of the stroke area in cases 1 (initial ASL images) and 2 (follow-up ASL images). It is interesting to note that, in case 1 , this area corresponded to an area of less diffusion abnormality. Collateral arteries may have contributed to perfusion within this region, which might have preserved some of the tissue. Arterial transit artifacts visualized within this region represent delayed arrival of transit, which supports the hypothesis of collateral perfusion. In adults, similar findings have been described and they are thought to be related to a better prognosis (5). In one of the cases described in the article by Wintermark et al. (10), hyperperfusion was visualized in the stroke area. Hyperperfusion has been shown previously with ASL MRI in adult stroke and is thought to be related to poststroke luxury perfusion. In our study, hyperperfusion within the stroke area was visualized in case 4. Measurements of volume flow using PC-MRA were congruous with luxury perfusion because a higher flow toward the ipsilateral hemisphere was measured. Higher-volume flow toward the affected hemisphere has earlier been demonstrated by van der Aa et al. (14) and was found in our study as well in case 1. In all our neonates, monitoring with NIRS showed an increased $\mathrm{rScO}_{2}$ in the affected hemisphere as compared with the contralateral hemisphere. This suggests as well that the local cerebral perfusion exceeded the metabolic demands in the affected hemisphere. Although higher $\mathrm{rScO}_{2}$ was a general finding, hyperperfusion was not visualized in all cases. As such, this increased $\mathrm{rScO}_{2}$ in cases without hyperperfusion may be attributed to the attenuated oxygen utilization in injured or dead tissue, whereas in case 4 hyperperfusion may contribute to increased $\mathrm{rScO}_{2}$. Interpretation of $\mathrm{rScO}_{2}$ values after PAIS has to be handled with care because the relationship between $\mathrm{rScO}_{2}$ values in the affected hemisphere and outcome might be dual. Earlier studies have shown a relation between hyperperfusion and improved neurodevelopmental outcome 


\section{Articles $\mid$ De Viset al.}

$(15,16)$, and as such in cases with hyperperfusion, increased $\mathrm{rScO}_{2}$ in the affected hemisphere might be beneficial. On the other hand, an increase in $\mathrm{rScO}_{2}$, typically starting $24 \mathrm{~h}$ after the event and evolving over the next $24 \mathrm{~h}$, has been proven to correlate with poor outcome (17). Dedicated ASL perfusion MRI studies should evaluate the influence of increased $\mathrm{rScO}_{2}$ values in combination with hyperperfusion vs. increased $\mathrm{rSCO}_{2}$ values without hyperperfusion on neurodevelopmental outcome. In addition, we want to mention that hyperperfusion has been visualized in an epileptogenic focus during epileptic activity (18). However, we consider this unlikely to be the cause of hyperperfusion because aEEG monitoring was performed in close proximity to MRI and did not reveal any remaining epileptic activity. In addition, monitoring during MRI examination did not show any abnormalities.

Three of four neonates in our study were born by emergency cesarean section due to fetal distress, a known risk factor for the development of PAIS (19). In those three neonates, some degree of global hypoxia-ischemia might have influenced our results. However, we can exclude severe hypoxia-ischemia because at most a discontinuous background pattern was seen in the affected hemisphere. In addition, global hypoxia-ischemia can be excluded because a normal voltage with superimposed sleep-wake cycling was present in the contralateral hemisphere of all neonates.

A strength of this study as compared with the previously performed ASL study in PAIS patients (10) is the evaluation of follow-up ASL MR images. When evaluating these images and comparing them with the initial ASL MR images, one should keep in mind that whole-brain perfusion increases. This is related to brain maturation and has been shown earlier in xenon-enhanced computed tomography (Xe-CT) studies (20), positron emission tomography studies (21) and ASL MRI studies (6). For illustrative purposes, whole-brain perfusion has been measured on all ASL images. As such, the increased brain perfusion with age can be appreciated by the reader. In three infants (cases 1, 3, and 4), follow-up MRI was performed after sufficient time to evaluate perfusion changes over time in patients with PAIS. In these three patients, perfusion within the stroke area recovered partly as compared with initial ASL images. Furthermore, the area of lower perfusion at this time point corresponded with the stroke area as visualized on the T2-weighted images. It should be noted that our perfusion measurements are lower than values earlier established with $\mathrm{Xe}-\mathrm{CT}$ and positron emission tomography (21). Whole-brain perfusion measured with Xe-CT was around $50 \mathrm{ml} / 100 \mathrm{~g} /$ min in infants aged $2-45 \mathrm{~d}(20)$ whereas we found an average value of $12 \mathrm{ml} / 100 \mathrm{~g} / \mathrm{min}$ in the scans performed at day 5 or 6. Although a maturational effect might contribute to this difference, the underestimation with ASL MRI can largely be attributed to the intrinsic low signal-to-noise ratio of an ASL experiment. This leads to loss of signal in the white matter. In addition, low brain perfusion in neonates increases this issue. A previous ASL MRI study (8) in neonates also demonstrated low perfusion values, $16.6 \mathrm{ml} / 100 \mathrm{~g} / \mathrm{min}$ in term-born infants imaged at term age. These values were slightly higher than ours. This can be attributed to different acquisition and quantification parameters. In their study, a pseudocontinuous labeling scheme-which increases the signal-to-noise ratio-was applied, whereas we used a pulsed labeling scheme. In addition, in our study-in order to achieve more accurate perfusion estimates - the longitudinal relaxation time of blood $\left(\mathrm{T}_{1 \mathrm{~b}}\right)$ was corrected for hematocrit (22), which tends to decrease the perfusion measurements based on an increased $\mathrm{T}_{1 \mathrm{~b}}$.

The major limitation of the current study is the small sample size, which does not allow us to make conclusions based on our findings. The low incidence of perinatal stroke complicates the acquisition of more data. As such, the main intention of this article is to show the possibilities ASL MRI holds and by itself stimulate other researchers to either conduct a multicenter study or to compile data from multiple centers. It is important to note that hemispheric perfusion values are shown only for illustrative purposes. We do not believe that these values support any hypothesis, not only due to the small sample size but mainly because interhemispheric differences of up to almost $20 \%$ have been demonstrated earlier with NIRS in subjects without stroke (23). To evaluate the additional value of hemispheric perfusion quantification, a case-control study should be performed. A second limitation is the different imaging parameters of the described sequences. For instance, the slice thickness of the DW images was $4 \mathrm{~mm}$, whereas ASL slices were $6 \mathrm{~mm}$ thick with a gap of $1 \mathrm{~mm}$ in between. In addition, different imaging plane angulations prohibit an exact comparison between the different sequences. In future studies scan sequences will be matched in order to enable an exact comparison between the different sequences. Overall, our results suggest that ASL MRI can be used to assess the infarct area. In the future, ASL MRI could be used to evaluate the role of luxury perfusion and arterial transit artifacts in the evolution of the infarct area. Furthermore, it might be used to assess the effect of future neuroprotective therapies (e.g., erythropoietin, hypothermia) on final infarct size.

In conclusion, ASL MRI can be used to visualize decreased perfusion, luxury perfusion, and arterial transit artifacts in perinatal arterial ischemic stroke patients.

\section{METHODS}

ASL perfusion MRI is part of the clinical MRI protocol in our institution. Infants admitted to our institution with the suspicion of PAIS were prospectively collected. In four infants, PAIS was confirmed on conventional MRI. The medical ethics committee of our institution approved the study without informed consent. MRI was performed on a 3.0 T Philips Achieva System (Philips Medical Systems, Best, The Netherlands). Images were acquired using the quadrature body coil for transmission and an eight-element phased-array SENSE head coil as a signal receiver. Initial MRI was performed 5-6 d after birth in all patients; follow-up MRI occurred $13 \mathrm{~d}$ to $16 \mathrm{wk}$ after the event. Infants were sedated prior to MRI, either with the administration of chloral hydrate through a nasogastric tube $(50-60 \mathrm{ml} / \mathrm{kg})$ or with an intramuscular injection of pethidine, chlorpromazine and promethazine. During MRI infants were wrapped into an MR-compatible vacuum cushion in order to prevent motion-related artifacts. Furthermore, for hearing protection MiniMuffs (Natus Europe, Münich, Germany) and earmuffs (EM's 4 kids, Everton Park, Australia) were used. Monitoring of heart rate, transcutaneous oxygen saturation, and respiratory rate was performed during MRI (Nonin Pulse Oxymetrie, Nonin Medical, 
Plymouth, MN), and a neonatologist was present throughout the entire MRI examination. The scan protocol for each patient consisted of conventional MRI, two-dimensional PC-MRA, diffusionweighted images, and ASL perfusion MRI. Scan parameters of the pulsed ASL (24) sequence were matrix $64 \times 64$, SENSE 2.5 , voxel size $3 \times 3 \times 6 \mathrm{~mm}$, gap between slices $1 \mathrm{~mm}$, TR/TE: $2,500 / 20 \mathrm{~ms}$, inversion time $1,500 \mathrm{~ms}, 70$ averages, labeling volume $150 \mathrm{~mm}, \mathrm{Q}_{2}$ TIPS (25) $600 \mathrm{~ms}$, and scan time 3:05 min. Perfusion was quantified on the $\Delta \mathrm{M}_{\text {total }}$ images using equation 2 in ref. 25. Quantification was based on the following assumptions; a longitudinal relaxation time of tissue of $1.5 \mathrm{~s}$ and a lambda of 0.9 . The fully relaxed magnetization of arterial blood was estimated from the data, and the $T_{1 b}$ was calculated on the basis of clinical hematocrit values (22). The hemisphere ipsilateral to the stroke area was denoted as the "ipsilateral" and the hemisphere contralateral to the stroke lesion as the "contralateral" hemisphere. Whole-brain perfusion and perfusion in the ipsilateral and the contralateral hemispheres were measured. In all patients, $\mathrm{rScO}_{2}$ was monitored bilaterally using NIRS in the frontoparietal region (INVOS 5100c; Somanetics, Troy, MI). $\mathrm{rScO}_{2}$ values obtained half an hour before and after MRI, measured in the ipsilateral and contralateral hemisphere, were averaged. Epileptic activity was diagnosed and evaluated with bilateral (C3-P3, C4-P4) aEEG tracing (BRM2 or BMR3 Brain Monitor, Natus, Seattle, WA). Only during MRI were the monitoring devices disconnected. The babies were treated sufficiently with antiepileptics before going for MRI. Insufficient treatment of epileptic activity was a contraindication for MRI.

\section{STATEMENT OF FINANCIAL SUPPORT}

This research was supported by the Dutch technology foundation "Stichting Technologische Wetenschappen", the Applied Science Division of The Netherlands Organization for Scientific Research (nederlandse organisatie voor wetenschappelijk onderzoek), and the technology program of the Ministry of Economic Affairs.

Disclosure: The authors have no conflicts of interest to disclose.

\section{REFERENCES}

1. Kirton A, deVeber G. Advances in perinatal ischemic stroke. Pediatr Neurol 2009;40:205-14.

2. Groenendaal F, Benders MJ, de Vries LS. Pre-wallerian degeneration in the neonatal brain following perinatal cerebral hypoxia-ischemia demonstrated with MRI. Semin Perinatol 2006;30:146-50.

3. Mercuri E, Barnett A, Rutherford $M$, et al. Neonatal cerebral infarction and neuromotor outcome at school age. Pediatrics 2004;113(1 Pt 1):95-100.

4. Bokkers RP, Hernandez DA, Merino JG, et al.; National Institutes of Health Stroke Natural History Investigators. Whole-brain arterial spin labeling perfusion MRI in patients with acute stroke. Stroke 2012;43:1290-4.

5. Chalela JA, Alsop DC, Gonzalez-Atavales JB, Maldjian JA, Kasner SE, Detre JA. Magnetic resonance perfusion imaging in acute ischemic stroke using continuous arterial spin labeling. Stroke 2000;31:680-7.

6. De Vis JB, Petersen ET, de Vries LS, et al. Regional changes in brain perfusion during brain maturation measured non-invasively with Arterial Spin Labeling MRI in neonates. Eur J Radiol 2013;82:538-43.

7. Licht DJ, Wang J, Silvestre DW, et al. Preoperative cerebral blood flow is diminished in neonates with severe congenital heart defects. J Thorac Cardiovasc Surg 2004;128:841-9.
8. Miranda MJ, Olofsson K, Sidaros K. Noninvasive measurements of regional cerebral perfusion in preterm and term neonates by magnetic resonance arterial spin labeling. Pediatr Res 2006;60:359-63.

9. Wang J, Licht DJ, Silvestre DW, Detre JA. Why perfusion in neonates with congenital heart defects is negative-technical issues related to pulsed arterial spin labeling. Magn Reson Imaging 2006;24:249-54.

10. Wintermark P, Warfield S. New insights in perinatal arterial ischemic stroke by assessing brain perfusion. Transl Stroke Res 2012;2:255-62.

11. Wintermark P, Hansen A, Gregas MC, et al. Brain perfusion in asphyxiated newborns treated with therapeutic hypothermia. AJNR Am J Neuroradiol 2011;32:2023-9.

12. Dullenkopf A, Kolarova A, Schulz G, Frey B, Baenziger O, Weiss M. Reproducibility of cerebral oxygenation measurement in neonates and infants in the clinical setting using the NIRO 300 oximeter. Pediatr Crit Care Med 2005;6:344-7.

13. van Bel F, Lemmers P, Naulaers G. Monitoring neonatal regional cerebral oxygen saturation in clinical practice: value and pitfalls. Neonatology 2008;94:237-44.

14. van der Aa NE, Porsius ED, Hendrikse J, et al. Changes in carotid blood flow after unilateral perinatal arterial ischemic stroke. Pediatr Res 2012;72:50-6.

15. Bivard A, Stanwell P, Levi C, Parsons M. Arterial spin labeling identifies tissue salvage and good clinical recovery after acute ischemic stroke. J Neuroimaging 2013;23:391-6.

16. Marchal G, Furlan M, Beaudouin V, et al. Early spontaneous hyperperfusion after stroke. A marker of favourable tissue outcome? Brain 1996;119 (Pt 2):409-19.

17. Toet MC, Lemmers PM, van Schelven LJ, van Bel F. Cerebral oxygenation and electrical activity after birth asphyxia: their relation to outcome. Pediatrics 2006;117:333-9.

18. Oishi M, Ishida G, Morii K, Hasegawa K, Sato M, Fujii Y. Ictal focal hyperperfusion demonstrated by arterial spin-labeling perfusion MRI in partial epilepsy status. Neuroradiology 2012;54:653-6.

19. Harteman JC, Groenendaal F, Kwee A, Welsing PM, Benders MJ, de Vries LS. Risk factors for perinatal arterial ischaemic stroke in full-term infants: a case-control study. Arch Dis Child Fetal Neonatal Ed 2012;97:F411-6.

20. Chiron C, Raynaud C, Mazière B, et al. Changes in regional cerebral blood flow during brain maturation in children and adolescents. J Nucl Med 1992;33:696-703.

21. Kinnala A, Suhonen-Polvi H, Aärimaa T, et al. Cerebral metabolic rate for glucose during the first six months of life: an FDG positron emission tomography study. Arch Dis Child Fetal Neonatal Ed 1996;74:F153-7.

22. Varela M, Hajnal JV, Petersen ET, Golay X, Merchant N, Larkman DJ. A method for rapid in vivo measurement of blood T1. NMR Biomed 2011;24:80-8.

23. Wijbenga RG, Lemmers PM, van Bel F. Cerebral oxygenation during the first days of life in preterm and term neonates: differences between different brain regions. Pediatr Res 2011;70:389-94.

24. Golay X, Petersen ET, Hui F. Pulsed star labeling of arterial regions (PULSAR): a robust regional perfusion technique for high field imaging. Magn Reson Med 2005;53:15-21.

25. Luh WM, Wong EC, Bandettini PA, Hyde JS. QUIPSS II with thin-slice TI1 periodic saturation: a method for improving accuracy of quantitative perfusion imaging using pulsed arterial spin labeling. Magn Reson Med 1999;41:1246-54. 